International Journal of Linguistics, Literature and Translation

ISSN: 2617-0299 (Online); ISSN: 2708-0099 (Print)

DOI: $10.32996 / \mathrm{ijllt}$

Journal Homepage: www.al-kindipublisher.com/index.php/ijltt

IJLLT

\title{
World War II and Its Impact on French Canadians
}

\author{
Daniela-Elena Duralia \\ Chercheur Postdoctoral, Université 1 Décembre 1918, Alba lulia, Roumanie
}

$\square$ Corresponding Author: Daniela-Elena Duralia, E-mail: d_duralia@yahoo.com

ARTICLE INFORMATION

Received: 14 October 2021

Accepted: 26 November 2021

Published: 30 November 2021

DOI: $10.32996 /$ ijllt.2021.4.11.19

\section{KEYWORDS}

Seconde Guerre mondiale, Français-canadiens, femmes, société, Québec.

\section{ABSTRACT}

This research takes the perspective of a Romanian-born philologist after having lived, studied, and taught in the Quebec public educational system for nearly 12 years. The main purpose of this study is to discuss the important influences World War II had on the evolution of Quebec society. Examining Quebec's social life and culture from a historic standpoint is a primordial step for immigrants and their integration into Quebec's society. An analysis of the corpus selected for this study, namely, Roch Carrier's La guerre, yes sir!, Jean Jules Richard's Neuf jours de haine, Jean Vaillancourt' $s$ Les Canadians errants, and Gabrielle Roy's Bonheur d' occasion, reveals that World War II marked in different ways the disturbance of the traditional, pastoral, and agrarian life, which triggered some modernist influences in people's lives. For instance, the war changed women's condition. When Canadian French men were shipped out to Europe to fight in the war, women were employed in Quebec's factories and plants. Even though they were paid less than men, they became independent and autonomous. Another example is the presence of British soldiers in Quebec, which disturbed the traditional lifestyle of French Canadians. It was difficult for English soldiers to understand the locals' culture, who in turn, perceived them as dangerous and responsible for the war. Nowadays, Quebecers' mentality is influenced by the various cultures they are surrounded by, yet, they still preserve some values which are originated during World War II.

\section{Introduction}

Cette recherche s'inscrit dans la perspective d'une philologue d'origine roumaine après avoir vécu, étudié et enseigné dans le système d'enseignement public québécois pendant près de 12 ans. Le but principal de cette étude est de discuter des influences importantes de la Seconde Guerre mondiale sur l'évolution de la société québécoise. Examiner la vie sociale et la culture du Québec d'un point de vue historique est une étape primordiale pour les immigrants et leur intégration dans la société québécoise.

Une analyse du corpus sélectionné pour cette étude, à savoir La guerre, yes sir! de Roch Carrier, Neufjours de haine de Jean-Jules Richard, Les Canadiens errants de Jean Vaillancourt et Bonheur d'occasion de Gabrielle Roy, révèle que la Seconde Guerre mondiale a marqué de différentes manières la perturbation de la vie traditionnelle et pastorale, ce qui a déclenché des influences modernistes dans la vie des gens. Par exemple, la guerre a changé la condition des femmes. Lorsque des hommes canadiens-français ont été envoyés en Europe pour combattre dans la guerre, les femmes ont été employées dans les usines du Québec. Même si elles étaient moins payées que les hommes, elles sont devenues indépendantes et autonomes. Un autre exemple est la présence de soldats britanniques au Québec, qui a bouleversé le mode de vie traditionnel des Canadiens français. Il était difficile pour les soldats anglais de comprendre la culture des locaux, qui à leur tour les percevaient comme dangereux et responsables de la guerre. De nos jours, la mentalité des Québécois est influencée par les diverses cultures dont ceux-ci sont entourés, pourtant, ils en conservent encore certaines qui demeurent des valeurs originaires de la Seconde Guerre mondiale.

\section{La revue de la littérature}

Dans les quatre romans, on remarque les influences de la guerre dans la vie des personnages en perturbant le silence. Dans le roman de Gabrielle Roy, la guerre amène le changement d'espace des Canadiens français. Ils n'avaient jamais voyagé à l'étranger. Le roman de Roy et celui de Carrier viennent compléter les tableaux de la société du Québec pendant la Deuxième Guerre mondiale

Copyright: (c) 2021 the Author(s). This article is an open access article distributed under the terms and conditions of the Creative Commons Attribution (CC-BY) 4.0 license (https://creativecommons.org/licenses/by/4.0/). Published by Al-Kindi Centre for Research and Development, London, United Kingdom. 
car ils sont centrés sur l'observation et l'analyse de l'individu et de la société, tandis que les deux autres romans présentent les terreurs de la guerre et les mémoires du vécu des combattants. Certains chercheurs comme Roxanne Bedard, Suzanne Bienvenu, Gilles Parent, Tara Beth Smithson, Pierre Nepveu ou Elisabeth Nardout-Lafarge ont traité ces quatre romans avec des perspectives différentes ayant des objectifs spécifiques. Quand même, aucune étude n'a observé ou analysé ces quatre œuvres avec l'objectif de clarifier la situation du peuple québécois et de l'évolution de la société selon les représentations dépeintes dans les quatre romans.

\section{3. Étude de cas}

Le contexte de la Seconde Guerre mondiale a provoqué des transformations dans les institutions culturelles et politiques surtout dans celles sociales. Le rôle de la femme était de soigner la famille et d'effectuer des travaux ménagers. En plus,

La puissance paternelle de l'homme chef de famille, par exemple, définit son rôle de gardien de l'honneur de la famille, à la fois au sein du foyer et, le cas échéant, devant les tribunaux. De même, les incapacités strictes des femmes mariées, des mineures et des interdits, si elles concernaient principalement la préservation du patriarcat et la protection des personnes vulnérables, devaient également empêcher que de dangereux canons lâches n'agissent au sein de la société en général au détriment de la famille (Reiter 2020: 99) (ma traduction)².

Le départ des hommes sur le front leur donne la liberté de se débrouiller. Les femmes commencent à être plus présentes dans la vie sociale. Dans le roman Bonheur d'occasion, Gabrielle Roy utilise la fresque sociale de la ville de Montréal, qui autrefois avait ses traditions, ses modes de vie et ses patois; la guerre a entraîné des éléments modernes dans la vie urbaine (importance des magasins, le cinéma, le mouvement, le chômage). Florentine, le personnage principale, veut faire partie de la société moderne de la ville de Montréal.

Dans le roman de Carrier, la guerre rend les femmes autoritaires. Amélie maltraite son mari, Henri, revenu du front. En plus, madame Joseph méprise son mari pour avoir refusé d'aller à la guerre, en le considérant comme lâche et manquant de masculinité, surtout après avoir coupé sa main pour ne pas être envoyé sur le front.

La guerre entraîne une valorisation de l'individu en tant que conscience autonome et indépendante. L'individu n'est plus dominé par le pouvoir clérical. Celui-ci prend une autre position dans la société. Dans Bonheur d'occasion, Emmanuel Mounier soutient le personnalisme en se montrant intéressé à participer à la création d'une pensée catholique au Québec.

L'esprit du personnalisme, de la liberté de l'individu, surtout les droits des femmes dans la société, ont connu un soutien important dans la société. Le ministère des Anciens, créé en 1944, supervisait le retour des soldats au Canada, les aidait à se trouver un emploi ou à faire des études. Le système fragile n'a sûrement pas protégé chaque individu, car le personnage de Vaillancourt est ridiculisé par l'engagé de l'institution pour ses efforts de s'inscrire à l'université.

Aujourd'hui, l'individu est protégé par des lois dans tous les instituts du Québec.

En plus, la pratique de la religion n'est plus imposée. Reiter informe que plus tard dans le XIXe siècle, les droits de l'individu ont commencé à apparaître dans le contentieux de certaines questions qui chevauchaient le droit public et le droit privé. La liberté religieuse était un exemple précoce et important [...] (Reiter $2020: 300)$ (Ma traduction)3.

L'esprit des Canadiens français change une fois la guerre commencée. Dans le roman de Jean-Jules Richard, le gradé canadienfrançais est déstabilisé par la réalité qu'il vit. Il s'écroule en se mettant sur la ligne de front, incapable de réaliser dans quel danger il met ses subordonnés.

L'individu canadien-français est attaché à son espace qui pendant cette période est envahi ou changé. Le sentiment d'appartenance à la naissance d'une identité canadienne-française est remarqué par plusieurs écrivains canadiens-français, parmi lesquels Marie de I'Incarnation note « [...] il n'y a rien ce me semble sous le ciel, qui soit capable de m'ébranler n'y de me faire sortir de mon centre, c'est ainsi que j'appelle le Canada » (Marie de l'Incarnation 1971: 569).

\footnotetext{
2 "The male head of household's puissance paternelle, for example, defined his role as guardian of the family's honour, both within the home and, if necessary, before the courts. Similarly, the strict incapacities of married women, minors, and those under interdiction, while mainly about the preservation of patriarchy and the protection of the vulnerable, were also to prevent dangerous loose cannons from acting up within society at large to the detriment of the family" (Reiter 2020: 99).

3 "Later in the nineteenth century, the rights of the individual began to appear in the litigation of certain issues that straddled public and private law. Religious liberty was an early and important example [...]" (Reiter 2020: 300).
} 
Spécifiquement à la vie traditionnelle, en général, les hommes doivent quitter leurs maisons. Selon Mircea Eliade, le chaos et le cosmos :

caractérise[nt] les sociétés traditionnelles, c'est l'opposition qu'elles sous-entendent entre leur territoire habité et l'espace inconnu et indéterminé qui l'entoure: le premier, c'est le « Monde » (plus précisément: « notre monde »), le Cosmos; le reste, ce n'est plus un Cosmos, mais une sorte d'« autre monde », un espace étranger, chaotique, peuplé de larves, de démons, d'étranger » (assimilés, d'ailleurs, aux démons et aux fantômes). (Eliade 1957: 32)

De plus, Frederick Karl note que quand:

un état séculier se transforme, momentanément au moins, en une expérience transcendantale. Et quand cela est perdu, tout est apparemment perdu innocence, esprit, même force physique. La perte de ce sentiment sacré, ou le sentiment qu'il ne peut pas être assimilé dans nos vies crée chez les écrivains d'après-guerre un terrible affaiblissement (Karl 1983: 43) (Ma traduction) ${ }^{4}$.

La guerre, yes sir! de Roch Carrier utilise la parodie pour mettre encore plus en évidence l'injustice du déracinement des soldats arrachés de leurs nids édéniques et envoyés dans l'enfer. De plus, leurs sacrifices ne sont même pas admis par les membres de leur famille. La situation d'Henri est exagérée, car il est culpabilisé par Amélie, sa femme pour avoir perdu sa place en tant que mari et père à cause de sa participation à la guerre.

-Pourquoi t'ont-ils renvoyé ici?

-Je suis fatiguée. C'est fatigant la guerre.

-Pendant que tu es ici, qui va se battre contre les Allemands? (Carrier 2006: 6)

Tandis que le célibataire, Arthur, I'amoureux de sa femme, qui a pris sa place dans sa propre famille et sa maison, lui dit :

-Tu es un soldat...

-Des Allemands! Je n'ai jamais vu hostie d'Allemand.

-Tu es un soldat, tu as l'uniforme, les bottes; moi, je suis un fermier, et un père de famille; j'ai deux couples de jumeaux et Amélie grossit encore. (Carrier 2006: 7)

Bérubé est un autre soldat revenu de la guerre qui dévoile ses souffrances par sa réaction aux moqueries d'un paysan:

Tu trouves qu'on s'amuse à la guerre? [...].Je vais te faire comprendre ce qu'est la guerre [...] Pendant qu'il éructait ses blasphèmes, Bérubé frappait Arsène au visage [...] non pas du poing, mais de sa main ouverte, et le gros visage $d^{\prime}$ Arsène, se tordait sous la douleur. Bérubé avait les yeux rouges, la grosse tête d'Arsène se balançait selon les coups[...], le veston était en pièces, la chemise déchirée, Bérubé aboyait. (Carrier 2006: 44)

Bérubé ne pouvait plus crier, ni blasphémer, ni parler. Une colère aiguë l'étreignait à la gorge, ses yeux brûlaient, il éclata comme un enfant en sanglots. (Carrier 2006: 45)

La technique que l'auteur emploie est décrite comme :

Un mélange des formes et des styles, brouillant obstinément la frontière qui sépare le comique et le sérieux, la parole brute et l'écriture sophistiquée, la tradition populaire et la culture lettrée. (Biron, Dumont, Nardout et Lafarge 2010: 472)

Cette technique l'aide à illustrer la fragilité émotionnelle et psychique, et aussi la simplicité, la pauvreté, le manque de culture des paysans qui avaient été heureux dans leur communauté, où ils se réjouissaient de la pratique de leur tradition populaire.

Dans Neuf jours de haine, les soldats canadiens ne se sentent pas en sécurité dans la ville. Ce n'est pas le combat qui attriste; c'est surtout l'atmosphère de guerre qui est mise en évidence pour souligner l'état d'esprit des hommes. « Des soldats portent encore les pantalons et le kepi verts. Ils circulent aussi désabusés que les étrangers. » (Richard 1999: 357) Le caporal, Martedale, des

\footnotetext{
4 "A secular state is transformed, momentarily at least, into a transcendental experience. And when that is lost, all is apparently lost innocence, spirit, even physical strength. The loss of that sacred feeling, or the sense that it cannot be assimilated into our lives, creates in the postwar writers a terrible debilitation" (Karl 1983: 43).
} 
Maritimes, étudiant en génie civil, perçoit les citadins « avide[s] » parmi lesquels « [il] doit se battre pour servir son repas. Et les soldats doivent s'infiltrer pour se faire servir. » (Richard 1999: 293)

La détresse de la guerre prévaut et enveloppe la ville et les soldats à la fois. Jean-Jules Richard envisage une telle atmosphere dans plusieurs episodes dans son roman:

De vieilles maisons centenaires dardent la nostalgie autour d'elles. Des bouts de rue de pierres rondes usées par le temps font vaciller dans la marche. Les rues sont désertes, grises et tristes. Le monde entier a les reins cassés. Le cafard nage à travers les rues sur le sens de la longueur. Le spleen bloque les rues sur le sens de la largeur. [...] L'oxygène lui-même encore imbu de la fluidité de la cinquième dimension suinte la mélancolie et le deuil. (Richard 1999: 357-358)

D'une manière différente, Jean Vaillancourt, l'auteur de Les Canadiens errants, dépeint le paysage du village hollandais, en révélant la beauté de la nature qui est animée par un soldat. La « tranquillité grave » et « des fumées minces » attristent le paysage naturel du village. Même l'environnement semble être l'ennemi des soldats.

C'est le crépuscule. Une neige légère et une tranquillité grave descendent sur la colline. Des fumées minces et droites montent vers le ciel, entre les sapins droits et sombres. Un sentier à travers les sapins et les quelques bouleaux. Un garçon y monte depuis dix minutes, un soldat. II porte un uniforme kaki élimé, mais aux plis rigidement pressés. II a une taille moyenne avec des épaules minces, mais larges et arrondies qui tendent bien là. (Vaillancourt 1954: 45)

Dans le roman de Carrier, l'arrivée des soldats Canadiens anglais perturbe le silence des villageois Canadiens français. L'auteur clarifie aussi la nécessité de l'intervention des Canadiens anglais qui a été très importante dans l'évolution de la société francophone au Québec.

\section{Conclusion}

En dépit de la déstabilisation des individus, le départ des hommes sur le front représente une porte ouverte vers d'autres civilisations ainsi que le besoin que le personnage principale du roman de Roy, Florentine Lacasse ressente à l'idée, de faire partie de la vie de Montréal. La guerre a aussi donné cours à une escapade vers la liberté du mariage, des contraintes imposées, de recherche d'identité. L'environnement du village de ferme, entouré par la forêt, et la présence de la neige dans le roman de Carrier, contraste avec les éléments modernes (cinéma ou magasins) dans la ville de Montréal du roman de Roy.

Les femmes ont de plus en plus de soutien pour se débrouiller dans la vie sociale, aujourd'hui en bénéficiant des meilleurs droits légaux sur tous les plans sociaux. Pour n'importe qu'elle religion ou pratique, l'individu québécois d'aujourd'hui réjouit un système qui encourage l'essor professionnel et celui individuel à la fois. La famille, comme telle est également protégée, mais chaque membre jouit de ses droits à lui, soit les enfants, soit les adultes.

Aujourd'hui, l'endroit ne représente plus un repère sécuritaire pour l'espace personnel. Le peuple du Québec continue de voyager, de changer de domicile sans être aussi bouleversé émotionnellement ou psychiquement. II a appris à vivre et à accepter toutes sortes de nationalités. C'est juste en connaissant les différences qui distinguent les individus qu'on peut se respecter mutuellement.

Funding: This research received no external funding.

Conflicts of Interest: The authors declare no conflict of interest.

\section{References}

[1] Reiter, E. H. (2020). Wounded Feelings, The Osgoode Society for Canadian Legal History, University of Toronto Press. 99

[2] Reiter, E. H. (2020). Wounded Feelings, The Osgoode Society for Canadian Legal History, University of Toronto Press. 300

[3] De I'Incarnation, M. (1971). Correspondance, Guy Marie Oury (éd.), Solesmes, Abbaye Saint-Pierre, 1971, Lettre CLXIX, De Québec, à une Ursuline de Tours, fin 1655,. 569

[4] Eliade, M. (1957), Le sacré et le profane, Gallimard, France. 32

[5] Karl, F. R. (1983). American Fictions 1940-1980, Harper \& Row, Publishers, New York. 43

[6] Carrier, R. (2006). La guerre, yes sir!, Bloomsbury Publishing PLC, Bristol Classical Press, Londres, .6

[7] Carrier, R. (2006). La guerre, yes sir!, Bloomsbury Publishing PLC, Bristol Classical Press, Londres,. 7

[8] Carrier, R. (2006). La guerre, yes sir!, Bloomsbury Publishing PLC, Bristol Classical Press, Londres,. 44

[9] Carrier, R. (2006). Carrier, La guerre, yes sir!, Bloomsbury Publishing PLC, Bristol Classical Press, Londres. 45

[10] Biron, M. F. D. (2010) Histoire de la littérature québécoise, Les Éditions du Boréal, Canada, 472

[11] Richard, J (1999). Neufjours de haine, Bibliothèque québécoise, Canada. 357

[12] Richard, J. (1999). Neuf jours de haine, Bibliothèque québécoise, Canada, 293

[13] Richard, J. (1999). Neuf jours de haine, Bibliothèque québécoise, Canada. 357-358

[14] Vaillancourt, J. (1954). Les Canadiens errants, Le Cercle du livre de France. 45 